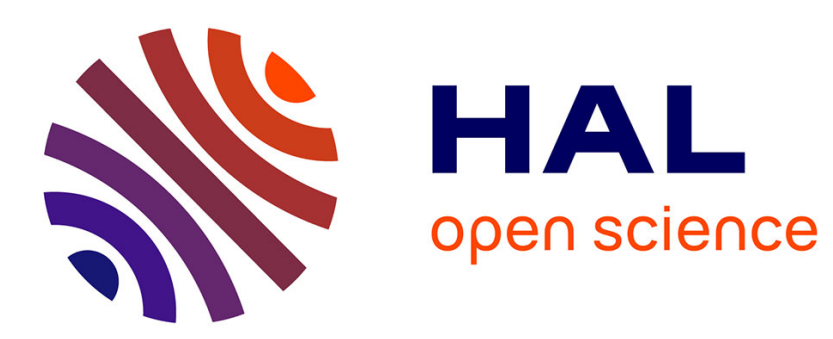

\title{
Le café en Amérique latine, une durabilité à géométrie variable \\ Jean-Christian Tulet
}

\section{To cite this version:}

Jean-Christian Tulet. Le café en Amérique latine, une durabilité à géométrie variable. Géocarrefour - Revue de géographie de Lyon, 2008, Agricultures, durabilité et territoire, 83/3, pp.171-180. 10.4000/geocarrefour.6845 . hal-02562280

\section{HAL Id: hal-02562280 \\ https://hal.science/hal-02562280}

Submitted on 4 May 2020

HAL is a multi-disciplinary open access archive for the deposit and dissemination of scientific research documents, whether they are published or not. The documents may come from teaching and research institutions in France or abroad, or from public or private research centers.
L'archive ouverte pluridisciplinaire HAL, est destinée au dépôt et à la diffusion de documents scientifiques de niveau recherche, publiés ou non, émanant des établissements d'enseignement et de recherche français ou étrangers, des laboratoires publics ou privés. 


\section{Géocarrefour}

Vol. 83/3 | 2008

Agricultures, durabilité et territoire

\section{Le café en Amérique latine, une durabilité à géométrie variable}

Coffee cultivation in Latin America: sustainability of 'a variable geometry'

Jean-Christian Tulet

\section{CpenEdition}

1 Journals

Édition électronique

URL : http://journals.openedition.org/geocarrefour/6845

DOI : 10.4000/geocarrefour.6845

ISSN : 1960-601X

Éditeur

Association des amis de la Revue de géographie de Lyon

Édition imprimée

Date de publication : 1 septembre 2008

Pagination : 171-180

ISSN : 1627-4873

\section{Référence électronique}

Jean-Christian Tulet, « Le café en Amérique latine, une durabilité à géométrie variable », Géocarrefour

[En ligne], Vol. 83/3 | 2008, mis en ligne le 01 octobre 2011, consulté le 03 mai 2019. URL : http:// journals.openedition.org/geocarrefour/6845; DOI : 10.4000/geocarrefour.6845

(c) Géocarrefour 
Jean-Christian TULET

GEODE Toulouse

\section{MOTS CLÉS}

Caféiculture, paysannerie, durabilité, Amérique latine, colonisation

\section{KEY WORDS}

Coffee production, peasantry, sustainability, Latin America, colonization

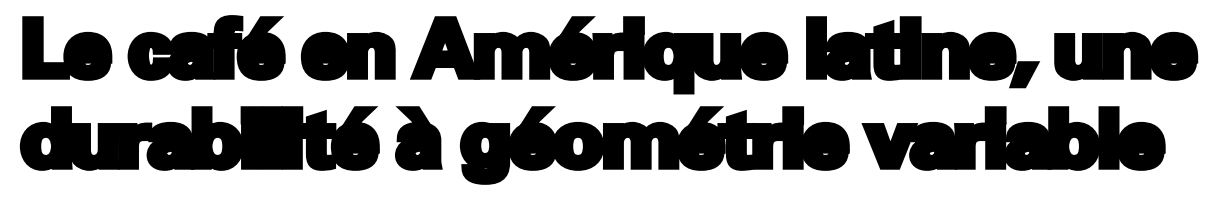

RÉSUMÉ

En Amérique latine, le café peut être considéré comme le pire ou parmi les meilleurs des systèmes de production agricole, tout au moins au niveau de ses conséquences environnementales. Conduit dans le cadre de grandes exploitations au Brésil, tournées vers la recherche du profit le plus élevé et le plus immédiat, sa culture a abouti à la destruction d'espaces immenses. Par contre, la mise en exploitation de milieux montagnards potentiellement plus fragiles dans les cordillères hispanoaméricaines, par des populations paysannes soucieuses avant tout de fonder une exploitation agricole, n'a pas entraîné de dégradation majeure, bien au contraire, malgré un peuplement important de ces milieux. L'exemple de la caféiculture souligne donc la part des déterminations initiales et des orientations socio productives dans la durabilité du système de production agricole.

\section{ABSTRACT}

In Latin America, the production of coffee can be considered as the worst or among the best agricultural systems, at least in terms of environmental consequences. Grown on big farms in Brazil, designed to generate immediate and the highest profits, its cultivation has resulted in the destruction of large areas. However, the exploitation of potentially more fragile mountainous areas in the Hispanic-American cordillera, by a farming population preoccupied by setting up an agricultural holding, has not led to a major degradation of the environment - on the contrary; and this despite the existence of an important population in these areas. The example of coffee cultivation underlines the role played by initial determination and socio-productive orientations in the durability of the agricultural production system.

Par ses conséquences environnementales, la production caféière connaît un statut tout à fait particulier en Amérique latine, étant considérée comme la pire ou la meilleure des agricultures, selon le mode de production utilisé. Au Brésil, pays où la grande propriété a longtemps exercé une position dominante, elle a été perçue comme une activité de type minier, les grandes exploitations, les fazendas, se déplaçant au fur et à mesure de l'épuisement des terres. En revanche, dans tout le reste de l'Amérique latine, la même activité, sous sa forme traditionnelle, avant I'arrivée des variétés à haut rendement, est reconnue comme l'exemple même d'une activité " durable ", particulièrement recommandable dans le cas de milieux difficiles, telles les cordillères montagneuses. Elle est d'autant plus " durable " que ces exploitations, paysannes le plus souvent, ont fait depuis longtemps la preuve de leurs capacités de résistance face aux crises récurrentes qui secouent le marché caféier.

Dans tous les cas, on se situe dans un milieu intertropical, la caféiculture ne pouvant pas se déployer sous d'autres climats. L'opposition entre les deux systèmes ne peut donc pas être imputée à des différences de potentialités du milieu physique. Bien au contraire, dans le cas du Brésil, la caféiculture pauliste a beaucoup apprécié la fameuse terra roxa, la terre violette, dont la fertilité était légendaire, avec parfois des sols de plusieurs mètres d'épaisseur, d'une exceptionnelle richesse. Cela n'a pas empêché leur détérioration parfois.

Une même spéculation provoque donc des effets parfaitement opposés. L'exemple du café permet ainsi de souligner la responsabilité de l'action humaine dans la conservation ou la dégradation des milieux.

\section{UNE ACTIVITÉ PRÉDATRICE AU BRÉSIL}

Le système traditionnel de la fazenda : tout pour le profit

On visite aujourd'hui les vieilles bâtisses qui se trouvaient au cœur du système caféier latifundiste brésilien d'avant les années 1930. Beaucoup sont menacées de disparition, perdues au milieu des broussailles, avec leurs toits crevés ou déjà totalement effondrés. D'autres font I'objet de programmes de sauvetage, elles sont entrées dans des circuits touristiques, certaines même sont reconverties dans l'hôtellerie, avec parfois en prime culturelle de petites représentations théâtrales évoquant les heures de gloire de la fazenda. Mais du café, il n'en existe plus du tout: le pâturage domine aujourd'hui sur toutes les terres des alentours, lorsque l'élevage est encore possible. Ces vieilles régions caféières donnent I'apparence de milieux ruinés, peu occupés, où l'activité agricole ne se peut désormais se décliner que sous une forme très extensive.

Cette dégradation de terroirs autrefois fertiles est liée à leur usage totalement indiscriminé en faveur d'une caféiculture par de grands entrepreneurs exclusivement tournés vers le profit immédiat, sans aucune considération pour ce milieu naturel. Les dégâts provoqués par un système aussi peu économe des ressources naturelles ont été perçus depuis longtemps. Ils sont soulignés dans l'œuvre majeure de Pierre Monbeig : " Ce qui est particulièrement grave, c'est que, pendant de nombreuses années, en dépit des recommandations prodiguées par les techniciens, les défricheurs n'ont rien fait, à de très rares exceptions près, pour enrayer cette marche vers le désastre. Lorsque les cultures donnaient des signes trop évidents de fatigue, on les abandonnait : c'est la clé de la marche pionnière" (Monbeig, 1952, p. 79).

Dans sa conclusion, le grand géographe condamne sans appel ce système d'exploitation : "Si l'on tente de dresser le bilan de la marche pionnière sur les plateaux occidentaux de São Paulo et ceux du Nord du Parana, I'œuvre destructrice des pionniers semble considérable : destruction de la forêt et, du coup, destruction de la terre. Le ressort de la marche vers l'Ouest tient dans le désir tenace du gain. Pour le satisfaire, il faut d'abondantes récoltes de produits vendus et exportés au-delà des mers. L'économie du monde 
pionnier a imposé une technique agricole dévastatrice à ces hommes trop pressés. Elle a refoulé ce respect de la terre qui est le propre des paysans. Pour conserver les abondantes récoltes et pour continuer de vendre à travers le monde, les pionniers sont contraints à une course ininterrompue. Et là où sont passés les planteurs, I'herbe repousse mal» (Monbeig, 1952, p. 362).

On rencontre ce thème de la destruction tout au long de son ouvrage : " la marche vers l'Ouest... une dévastation sans fin " (p. 63), " détérioration radicale des sols " (p. 77), " œuvre dévastatrice " (p. 78). Pourtant à côté de ces jugements définitifs, l'auteur donne à l'occasion l'impression d'accorder des circonstances atténuantes aux planteurs paulistes, puisque cette destruction est conçue comme une fatalité propre aux pays tropicaux : " les procédés sont identiques dans tous les pays tropicaux et on a déjà décrit la technique dévastatrice du caboclo que le pionnier moderne a entièrement adoptée " (p. 221). Pour donner plus de poids à une affirmation aussi radicale, il cite des travaux de P. Deffontaines (Deffontaines, 1940 p. 45) et de P. Gourou (Gourou, 1947, p. 1), pourtant futur auteur des " terres de bonne espérance " (Gourou, 1982)... Ailleurs, une certaine nostalgie de l'âge d'or de la fazenda semble transparaître lorsque, après la crise de 1930 l'auteur donne l'impression de regretter l'accession à la propriété des "petites gens " et une orientation nouvelle vers la polyculture : " la frange pionnière ne connaît plus cette homogénéité qui, pendant les trois quarts de siècle où le café fut le roi incontesté, avait été le gage d'une stabilité aujourd'hui évanouie" (Monbeig, 1952, p. 295).

\section{Tout pour la monoculture du café}

Dans cette dernière référence, $P$. Monbeig évoque la grande période de la fazenda caféière, où tout était organisé autour de la seule activité caféière. Si le propriétaire acceptait l'installation de cultures intercalaires pendant les premières années de plantation, celles-ci étaient soigneusement réglementées pour qu'elles ne puissent pas nuire au supposé bon développement des caféiers. Dès les premières récoltes de café, elles étaient rapidement éliminées, quitte à accorder un lopin de terre aux colons pour qu'ils puissent cultiver ce dont ils avaient besoin pour se nourrir. Toute diversité culturale était même parfois prohibée par principe : " le contrat de colonat fixe la somme que l'immigré engagé comme colon doit recevoir pour prendre soin de 1000 pieds de café et le salaire qui lui sera payé par journées de travail fournies en surplus (...). II indique en outre ou bien que le colon est autorisé à faire des cultures vivrières intercalaires, ou bien qu'une certaine surface de terrain lui est remise pour sa libre disposition, mais à l'écart du cafezal » (Monbeig, 1950, p. 140).

Les systèmes de cultures ne sont pas nécessairement les thèmes les plus développés par I'auteur, tout au moins par rapport à la dynamique générale du front pionnier. Ces systèmes sont évoqués assez tardivement dans le texte, lorsque sont abordés les changements dans la propriété de la terre et que l'hégémonie de la fazenda a été mise à mal. C'est dans ce cadre, après d'ailleurs avoir rappelé que pour lui la supériorité des fazendas lui apparaissait certaine, en particulier pour des raisons de capacités techniques, que l'auteur souligne que le cafezal continue d'être formé selon " des procédés anciens suivis dans la plupart des pays producteurs de café. Dans des trous quadrangulaires (covas) profonds de 30 à 50 centimètres, on plante plusieurs boutures de café de façon que leurs sommets ne dépassent pas la surface du sol. Ensuite ces covas, disposées en files bien tracées, séparées par des intervalles réguliers, sont recouverts d'un petit échafaudage en bois (aracupa) qui les protège à la fois des animaux et des rayons du soleil. Lorsque le caféier est suffisamment grand, au bout de dix-huit mois ou de deux ans, on le débarrasse de ce fragile abri et c'est alors seulement que l'œil découvre un cafézal $^{1}$ " (Monbeig, 1952, p. 241). La différence entre un petit producteur et un plus grand ne se situerait pas dans les systèmes techniques, mais seulement dans le plus faible entretien de la parcelle par le premier, qui laisse sur place des souches et des troncs d'arbre et qui nettoie le sol beaucoup moins régulièrement. Dans tous les cas, les investissements, tout particulièrement en travail, demeurent peu importants. On récolte au moindre coût, quitte à ne pas toujours cueillir seulement les meilleures cerises et à endommager les rameaux des arbustes ${ }^{2}$.

Selon Monbeig, la production monoculturale ne diminue pas après la crise de 1929, que ce soit dans les petites exploitations qui naissent de la disparition de beaucoup de fazendas ou dans les grandes propriétés qui se recréent plus au Nord. Le nouveau modèle pourtant se singularise par un usage plus diversifié de l'espace disponible, avec le développement du "quatuor de base ", maïs, riz, haricot, manioc, mais également avec la montée en puissance du coton et de la canne à sucre. Toutefois, les principes demeurent identiques. Si les sitiantes (petits exploitants, qui parfois sont en faire-valoir direct, mais sont le plus souvent des métayers) développent des cultures intercalaires dans le cafezal, l'idéal demeure une stricte séparation entre les diverses activités. Le café se maintient sur les meilleures terres, celles des versants bien exposés, à l'abri des coups de froid et disposant des meilleurs sols. Les autres cultures se distribuent sur le reste de l'exploitation, sans qu'il y ait compénétration des activités.

Il semble que le principe de la monoculture n'ait guère changé depuis, tout au moins dans le cadre des grandes exploitations, toujours dominantes. Une nouvelle phase d'expansion assez proche du modèle pauliste se développe à partir de 1945. Celle-ci provoque la mise en place de "l'Axe du Café ", autour des pôles régionaux de Londrina et Maringá. Le Paraná devient la région caféière la plus importante du pays au début des années
1 - Pour notre part, nous n'avons jamais vu utiliser ce système dans aucun des pays caféiculteurs visités, pas plus en Amérique qu'en Afrique ou au Viêt Nam. La pratique générale dans tous ceux-là est de faire germer les grains de café dans des pépinières, de placer les plantules dans des sachets remplis de terre lorsqu'elles atteignent quelques centimètres. Les plants sont ensuite mis en terre lorsqu'ils ont entre 10 et $20 \mathrm{~cm}$. Le planteur peut luimême effectuer ces diverses opérations ou acheter les plants à des exploitations spécialisées.

2 - Pratique de "el ordeño", la " traite ", en Amérique hispanophone : on tire sur le rameau en récupérant une bonne part de ses feuilles et toutes les cerises, quels que soient leur degré de maturation ou leur qualité. 
3 - Source : Faostat

4 - Traduction JC. Tulet.
1960, jusqu'aux gelées catastrophiques des années 1970, après lesquelles cette région se reconvertit dans la culture du soja. Les caféières se situent aujourd'hui majoritairement dans le Minas Gérais, à l'abri des gelées (Grandjean, Tulet, 2000). Les différences régionales seraient toutefois aujourd'hui beaucoup plus marquées, certaines régions traditionnelles présentant une structure assez émiettée et des systèmes polyculturaux, alors que d'autres, comme le Cerrado mineiro, comportent un nombre important d'exploitations de plus de 500 voire 1000 ou 2000 hectares en monoculture (Grandjean, 1999).

\section{LE RÈGNE DE LA PAYSANNERIE CAFÉIÈRE DANS LE RESTE DE L'AMÉRIQUE LATINE}

Malgré une forte réduction de la surface des caféières (largement compensée par une très forte hausse des rendements), le Brésil demeure le très grand pays producteur de café, le plus grand du monde. En 2005-2006, il produit à lui seul près de la moitié du café latino-américain ${ }^{3}$. Il dispose de structures de production parmi les plus puissantes : la seule coopérative de Coaxupé dans le Minas Gérais traite une quantité de café équivalente à celle de toute la production du Venezuela...

Cette domination brésilienne ne se retrouve pourtant pas au niveau de la société des caféiculteurs. On estime le nombre des producteurs brésiliens autour de 300000 , soit un effectif à peine plus important que celui du Mexique, qui produit huit fois moins de café. Les caféiculteurs hispanophones dominent donc très largement en nombre, avec des centaines et des centaines de milliers d'exploitants. Les sociétés qu'ils composent sont parmi les plus dynamiques et les plus solides du monde rural latino-américain (Tulet, 1998).

\section{Fonder une exploitation, un des objectifs premiers}

Les caféiculteurs hispanophones sont des petits producteurs en faire-valoir direct dans leur écrasante majorité. Pour beaucoup, l'obtention de ce statut se trouve très précisément à l'origine de la fondation de leur plantation, même si interviennent d'autres raisons (initiatives privées, parfois celles de l'État, ou bien simple résultante d'une conjoncture particulière). En Colombie, l'extraordinaire colonisation antioquienne est ainsi le produit d'une volonté d'investissement de la bourgeoisie et d'une recherche de terres nouvelles de la part des petits producteurs : "les diverses avancées de la frontière agraire témoignent de la continuité d'un élément particulier de la colonisation colombienne des cent dernières années: la symbiose des efforts privés de planification de la part d'entrepreneurs capitalistes très puissants et le caractère spontané des migrations paysannes à la recherche de terres et de travail, avec un arrière plan de relative passivité étatique" (Palacios, 1983) ${ }^{4}$.

En fait, cette colonisation colombienne, qui aboutit à la création d'un groupe de population aux caractéristiques très marquées, la société Paisa, n'est originale que par son ampleur. On rencontre des phénomènes analogues à l'origine de beaucoup de caféicultures, et pas seulement en Amérique latine (Tulet, 2007). Dans d'autres cas, l'action de l'État apparaît plus importante, ainsi au Salvador ou au Guatemala. Toutefois les créations à partir d'une initiative gouvernementale sont plus nombreuses dans le reste du monde tropical qu'en Amérique latine. L'extraordinaire croissance de la caféiculture vietnamienne est en relation étroite avec la libéralisation économique (le Do Moi) que ce pays expérimente à partir des années 1980 . Ce changement de politique a été mis à profit par des milliers de familles pour créer une exploitation agricole. Dans cette perspective, autrefois comme aujourd'hui, la meilleure manière d'affirmer des droits sur une terre supposée vacante est de fonder une plantation pérenne.

La caféiculture peut également monter en puissance à partir d'une situation politique tout à fait particulière. Celle du Venezuela s'est développée plus rapidement que dans d'autres pays latino-américains, parce qu'au milieu du XIXe $s$. une partie de la population des terres basses des Llanos a été obligée de fuir la guerre civile. Elle a trouvé partiellement refuge dans les cordillères andines et s'est assimilée aux populations montagnardes. Cet afflux de population et de main-d'œuvre a favorisé l'expansion du café, au point que le Venezuela en était devenu le deuxième exportateur mondial à la fin du $\mathrm{XIX}^{\mathrm{e}} \mathrm{s}$. (Salcedo-Bastardo, 1982).

Quelles que soient les raisons qui se trouvent à I'origine de nouvelles caféicultures, on rencontre toujours un point commun : dans tous les cas, ce sont les paysans qui défrichent des terres forestières de moyenne montagne, situées en gros entre 800 et 1500 mètres d'altitude, libres (ou supposées libres) de tout droit de propriété. La plantation de café est d'abord une opportunité de s'approprier un espace avec un minimum d'investissement, le travail de la famille étant largement considéré comme "gratuit ". Ces mêmes déterminations sont toujours à l'œuvre encore aujourd'hui, ainsi au Pérou et au Honduras.

\section{La mise en place d'une arboriculture}

Cette caféiculture paysanne a permis par ailleurs la combinaison d'un produit négociable, le café, avec d'autres ressources qui assurent un degré d'autosubsistance tout à fait appréciable. Cela n'a pas été le résultat d'un choix, mais d'une obligation. Les variétés traditionnelles de café arabica (celles de moyenne montagne) sont peu exigeantes, elles demandent seulement une défense contre les excès de l'insolation (Tulet, 1992), contrairement à ce que I'on rencontre dans plantations brésiliennes, même traditionnelles. Des bananiers sont donc plantés pour cela en même temps que le café. Leur croissance très rapide permet une protection efficace des seconds, mais contribue également à la subsistance 
familiale, directement ou indirectement, en procurant une alimentation aux animaux de bassecour (cochon compris !) et même parfois quelques têtes de gros bétail (cheval ou bovins). Pour les mêmes raisons alimentaires, le producteur sème également, sur la même parcelle, divers fruitiers (orangers, avocatiers, goyaviers, mais ces arbres ne doivent pas être trop nombreux, pour cause de frondaisons trop épaisses), des plants de manioc (là encore en quantités modérées, mais parce que le manioc épuise la terre), un peu de maïs, un peu de canne à sucre (pour le jugo de panela), et bien $d^{\prime}$ autres plantes. En même temps, sont mis en place les vrais " arbres d'ombre ", dont la croissance est beaucoup plus longue. Ils sont ceux qui procurent à la culture du café la protection la plus adéquate contre le soleil. Ce sont des espèces légumineuses, qui donc contribuent à fertiliser le sol. Ces espèces sont assez variées et changent selon les pays : Bucare (Erythrina spp.), Guamo (Inga spp.) appelé Chalum au Guatemala, où I'on utilise également le Gravilea (Gravilea robusta cunn.).

Il existe ainsi plusieurs étages de végétaux dans une caféière traditionnelle, les plants de café se trouvant souvent totalement dissimulés par le reste, au point qu'il n'est pas rare que le non averti puisse douter de leur présence. La caféiculture traditionnelle est une arboriculture. Elle est à I'image de bien d'autres que I'on rencontre également dans le monde tropical que ce soit en Amérique, en Afrique ou en Asie et qui sont parmi les systèmes les plus durables, les plus protecteurs de l'environnement.

La diversité des ressources disponibles sur un même espace assure un degré assez élevé d'autosubsistance, bien commode pendant les périodes récurrentes où le café se vend mal. Dans certains cas, une partie des ressources secondaires peut même procurer un revenu d'appoint, par exemple avec la vente des régimes de bananes, lorsque l'exploitation n'est pas trop éloignée d'une route et que les coûts de transport ne sont pas trop élevés. Dans la pire des situations, le planteur peut même abandonner l'entretien de sa caféière pour aller rechercher du travail à l'extérieur. Grâce à sa rusticité, le tipica, une des variétés traditionnelles de café arabica parmi les plus répandues, est capable de supporter cet abandon temporaire et reprendre de sa vigueur lorsque la parcelle est à nouveau nettoyée et à nouveau entretenue.

$\mathrm{Si}$, en dehors de quelques pays (Brésil, Guatemala, Salvador) la petite et la moyenne propriété familiale dominent dans la caféiculture, cela tient en grande partie à la souplesse de ce système de production traditionnel, particulièrement bien adapté aux possibilités de ceux ne disposant que de leur force de travail. II n'existe pas d'économie d'échelle dans la caféiculture, pour cause $\mathrm{d}^{\prime}$ absence totale de mécanisation ${ }^{5}$. Les frais de culture sont donc proportionnels à la surface. En faisant d'abord appel à la main-d'œuvre familiale, la gestion d'une petite ou moyenne plantation est ainsi beaucoup plus aisée qu'une grande. Cette dernière doit au contraire affronter de très difficiles problèmes de recrutement et d'administration de personnel, qui dans certains cas sont en mesure de la mettre en péril, lorsque les prix de vente du café ne permettent plus de faire face aux coûts salariaux. La grande exploitation caféière n'a ainsi dominé et ne domine réellement que dans les pays où les planteurs ont pu exercer, au moins pendant une période historique décisive, un contrôle étroit sur cette main-d'œuvre, soit par contrainte (esclavage au Brésil, travail forcé au Guatemala), soit par des formes très particulières de recrutement (organisation de l'immigration au Brésil, comme solution à la suppression de I'esclavage, avec également comme conséquence le souci de limiter le plus possible l'investissement travail, ce qui provoque diverses conséquences dans la qualité finale du produit). Mais dans tous les cas, les périodes de crise ont toujours vu s'accroître la part des exploitations paysannes aux dépens des grandes exploitations (Tulet, 1997).

\section{Un système traditionnel durable et reproductible}

Cette plasticité a assuré le succès de la caféiculture dans nombre de pays hispanophones. Les moyennes montagnes tropicales étant relativement salubres, la forte natalité traditionnelle a provoqué une croissance très rapide de la population. La plupart des terroirs de café se sont très rapidement densifiés, ce qui a entraîné un puissant mouvement de colonisation de la moyenne montagne. Le plus bel exemple en est probablement celui du pays Paisa en Colombie, où toutes les Cordillères au Sud de Medellín ont été progressivement colonisées dans un mouvement qui a duré plus d'un siècle et qui ne s'est achevé que tout récemment. Aujourd'hui, ce pays Paisa est devenu une des articulations majeures du monde rural colombien, jouissant d'une relative tranquillité (compte tenu du contexte général du pays) et d'une relative prospérité, un des derniers remparts contre le chaos qui pourrait menacer.

En dépit de cette colonisation très active, les terroirs d'origine n'ont pas pour autant été abandonnés. Le système traditionnel du café dans le monde hispanique est un système durable au plein sens du terme. Une exploitation caféière peut se maintenir tout le temps de la vie active de celui qui la fonde et même au-delà. Un plant de café traditionnel dure des dizaines d'années : lors de visites, il n'est pas rare que l'on présente (avec fierté) des caféiers supposés avoir dépassé une durée de vie de plus d'un siècle. Bien entendu, leur rendement ne se situe pas parmi les plus performants. Mais une caféière dont les plants ont été régulièrement remplacés dure très longtemps: la fertilité demeure, grâce à la diversité végétale que I'on y rencontre, à l'importance de la litière ainsi générée, mais également grâce à la présence des arbres spécialisés dans d'ombrage et qui nourrissent le sol.
5 - Le seul exemple notable de mécanisation se rencontre aujourd'hui dans les cerrados brésiliens, avec des machines à récolter, très comparables à celles que I'on utilise pour la récolte du raisin. Cela suppose une topographie apte à leur usage, totalement incompatible avec celle des terroirs des autres pays producteurs. 
6 - On peut qualifier la mise au point de ces nouvelles variétés de "révolution verte", parce qu'il s'agit des mêmes procédures et des mêmes résultats que dans le cas des céréales : croisement de variétés existantes (il ne s'agit absolument pas d'OGM) avec comme résultat la mise au point de nouvelles variétés beaucoup plus productives, mais beaucoup plus exigeantes en traitements.
II existe toutefois de vieux terroirs avec de très mauvaises conditions d'exploitation, des rendements très médiocres et de gros problèmes de parasites, comme dans les vieilles régions caféières du Venezuela. Cette situation est souvent la résultante d'une succession mal assurée ou non assurée. Dans ce cas, le vieux producteur demeuré sur sa parcelle, souvent dans des conditions très médiocres, ou son successeur parti en ville, se contentent d'une économie de cueillette, avec très peu d'investissements, un entretien très rudimentaire, et des rendements très faibles, de quelques sacs. Dans ces conditions, la caféière $s^{\prime}$ apparente davantage à une friche, souvent assez difficile d'accès, qui permet tout juste de survivre à son détenteur, s'il ne dispose que de cette ressource. Le mauvais entretien retentit sur toute la petite région, comme foyer de propagation des parasites, d'autant qu'en général le vieillissement (des personnes et des plantations) constitue un phénomène régional. Cette situation touche avant tout les pays où I'agriculture en général et la caféiculture en particulier ont connu des difficultés majeures.

\section{L'ENTRÉE DE LA CAFÉICULTURE DANS L'ÈRE PRODUCTIVISTE}

II faut toutefois ajouter que la relative solidité du système traditionnel se paye par la faiblesse de l'ensemble des ressources et surtout par celle des rendements en café. Lorsque les exploitations se spécialisent, c'est-à-dire lorsqu'elles sacrifient une part de leur diversité, les rendements en café s'élèvent, mais ils restent en général modestes. Tout change avec l'adoption des nouvelles variétés dites hybrides, venues des centres de recherche brésiliens.

\section{Une révolution verte}

Ces dernières ont révolutionné la caféiculture mondiale depuis une trentaine d'années. II s'agit principalement, par ordre d'apparition, du caturre et du catuai. II en existe aujourd'hui bien d'autres, en voie de diffusion ou d'expérimentation. Ces nouvelles variétés ${ }^{6}$ sont souvent dites de soleil, parce qu'elles n'ont plus besoin d'être protégées contre celui-ci. La strate arborée n'est donc plus nécessaire. Le système de production et les paysages qui lui sont associés changent radicalement. Toutes ces nouvelles variétés ont en commun d'être plus fragiles et d'avoir des exigences culturales infiniment plus grandes que les traditionnelles. Elles disposent d'une durée de vie bien inférieure, huit ans environ, pouvant aller jusqu'à douze après recépage. Leur efficacité diminue ensuite fortement. Elles demandent surtout des soins et des entretiens beaucoup plus importants, avec des désherbages plus fréquents et diverses applications de pesticides, fongicides et d'engrais. La diversité végétale n'est plus nécessaire, elle peut au contraire s'opposer à un entretien efficace de la plantation. Par ailleurs, leur concurrence risque d'entraîner une diminution du nombre de caféiers à I'hectare. Avec la variété tipica, leur densité ne s'élevait guère au-delà de 1500 pieds/ha. Elle est au moins du double avec les nouvelles. II n'est d'ailleurs pas rare de rencontrer des parcelles avec plus de 5000 pieds, voire plus de 10000 pieds/ha. La physionomie des caféières s'en trouve radicalement changée. Au café-fouillis des parcelles traditionnelles succèdent les stricts alignements (très semblables à un vignoble) des nouvelles plantations.

Tout cela provoque un investissement en travail et des coûts bien plus élevés que dans le système traditionnel. Mais les avantages se situent à la hauteur des risques encourus. Les rendements d'une caféière traditionnelle restent le plus souvent très modestes, de l'ordre de la dizaine de sacs de $60 \mathrm{~kg}$. Ils sont en général beaucoup moins élevés, pour cause d'entretien déficient ou d'attaques de maladies diverses. Tout change avec les nouvelles variétés pour lesquelles un rendement de 30 sacs/ha constitue un minimum. II semble relativement aisé avec un peu de soins, d'obtenir une cinquantaine de sacs. On rencontre d'ailleurs de plus en plus fréquemment des exploitations avec des rendements atteignant et dépassant les 100 sacs/ha ! Avec quelques hectares un petit paysan peut donc aujourd'hui produire davantage qu'un grand propriétaire traditionnel. On peut toutefois imaginer ses réticences pour effectuer un changement aussi radical, avec l'ensemble des risques qui lui sont associés.

En fait, ces nouveautés se sont souvent avérées attractives. Très rapidement, le problème pour les producteurs n'a plus été d'évaluer les mérites respectifs de chaque système, mais de trouver les moyens d'abandonner l'ancien pour adopter le moderne. En dépit de tous les problèmes qu'il pouvait poser (en particulier travail et gestion du temps de travail plus délicats), la croissance considérable a permis une hausse appréciable du niveau de vie de l'exploitant. En conséquence, à présent, il n'existe plus beaucoup de pays producteurs latino-américains où la caféiculture ne se soit pas déjà radicalement transformée par l'adoption de ces nouvelles variétés.

\section{Les risques engendrés par le nouveau modèle}

Un tel modèle suscite les mêmes réactions que pour les autres formes d'agriculture productiviste. On peut les rassembler selon deux thèmes, une plus grande vulnérabilité du petit producteur, une aggravation majeure des risques écologiques, allant jusqu'à mettre en cause la durabilité du système.

Un des arguments souvent employés contre la révolution verte, contre l'intensification de l'agriculture au moyen de semences ou de plants à haut rendement, est que cette adoption ne peut être que sélective et qu'elle renforce les catégories de producteurs les plus aisées. Les riches deviendraient plus riches et les pauvres plus pauvres. Une grande quantité d'observations ont été effectuées à ce propos, tout particulièrement 
en Inde à propos de la céréaliculture. Bon nombre de leurs conclusions semblent indiquer que si les riches deviennent plus riches, les pauvres deviennent moins pauvres, grâce à une diffusion finalement assez large de l'usage de ces semences, mais également parce que leur application demande plus de travail, donc davantage de salaires versés et même une tendance à l'augmentation de la rémunération salariée. II existe sur ce point beaucoup moins d'analyses concernant la caféiculture. On peut imaginer que les mêmes causes produisent les mêmes effets, sauf dans le cas de la mécanisation de la récolte, telle qu'elle s'est développée dans le cas très particulier des grandes plantations du Triangulo mineiro au Minas Gérais, où la topographie relativement plane permet cette adoption. Ailleurs, l'essentiel du travail dans la caféière étant toujours manuel, le passage aux variétés à haut rendement se traduit par un investissement plus important en termes de maind'œuvre, le plus souvent assuré par les disponibilités au sein de la famille. Mais ce n'est pas le cas pour la récolte, ce qui donne lieu à des déplacements de grande importance. Beaucoup de planteurs, dans beaucoup de pays, se plaignent d'ailleurs du manque de personnel occasionnel doté de la compétence nécessaire et de sa cherté.

Si la diffusion des variétés à haut rendement ne semble pas avoir provoqué une réelle modification dans le rapport entre riches et pauvres, il n'en est sûrement pas de même pour ce qui concerne les modifications culturales. II est difficile d'imaginer que la disparition plus ou moins complète des étages de végétation présents dans le modèle traditionnel et le recours bien plus massif aux intrants industriels n'entraîne pas diverses conséquences à plus ou moins long terme sur la qualité de l'environnement. Mais il est assez difficile d'en mesurer la réalité. Des analyses ont été effectuées afin d'évaluer les pertes en sol. Elles ne donnent pas toujours des résultats très probants. Le problème est surtout important entre deux cycles de plantation, lorsque les plants trop vieux ont été coupés ou recépés et que la parcelle reste à nu. Mais il y a souvent des cultures intercalaires qui occupent le sol pendant cette période. Par ailleurs, dès que les plants ont grandi, ils sont tellement nombreux que leurs frondaisons se touchent. Le sol est totalement couvert, ce qui limite les risques d'érosion.

La question des intrants industriels semble bien plus grave. Leur application risque d'entraîner un appauvrissement du sol, compensé par une augmentation des quantités utilisées et provoquant une élévation corrélative des coûts de production. On pourrait dans ce cas aboutir aux mêmes conséquences que pendant les périodes des fronts pionniers brésiliens, avec l'abandon de la caféiculture sur les terroirs concernés et sa substitution par des cultures beaucoup moins intensives. Toutefois, force est de constater que I'on en connaît assez peu d'exemples. Cela est peut-être lié à un manque de recul historique (il faut toutefois rappeler que ces nouvelles variétés sont apparues depuis plus d'une génération). Plus vraisemblablement, cela tient probablement à une relative rareté des parcelles en monoculture et en pleine exposition solaire, sauf dans quelques pays.

\section{Adaptations et diversifications}

Dans la pratique, les mutations s'effectuent en général progressivement, les caféières traditionnelles ne disparaissant que peu à peu à I'intérieur de chaque exploitation. D'autre part, les mutations ne sont pas toujours radicales. Les producteurs, en particulier les plus modestes, souhaitent le plus souvent conserver une partie de leurs bananiers et arbres fruitiers, en dépit des recommandations éventuelles de techniciens bien intentionnés. En conséquence, si la diversité végétale originelle diminue, elle ne disparaît pas toujours totalement. La plantation ne présentant que des alignements serrés de plants de café est loin de toujours constituer la norme.

Elle l'est d'autant moins que les techniciens préconisant une monoculture totale sont contredits par d'autres qui souhaitent une certaine diversité. Ainsi le Manuel du caféiculteur guatémaltèque recommande le maintien d'une protection arborée pour les nouvelles variétés, parce que celle-ci est supposée élever ou maintenir la qualité du café national, " le meilleur du monde " comme il se doit. Ce couvert végétal demeure donc très souvent.

L'ingéniosité des petits producteurs laisse d'ailleurs très loin derrière toutes les solutions proposées. Beaucoup d'entre eux cherchent toujours à diversifier leurs ressources, en particulier pendant les premières années de plantation, lorsque le caféier ne produit pas encore. Le strict alignement des nouvelles variétés (nécessaire à leur entretien) est alors mis à profit pour mettre en place des cultures intercalaires: maïs et arracacha (un tubercule, le maïs est d'abord récolté, ensuite l'arracacha, plus long à venir à maturité) ou manioc et maïs (même scénario). D'autres se lancent pendant les deux ans d'attente avant la première récolte de café dans la culture de la tomate, du poivron ou des haricots verts, toujours dans les allées séparant les alignements de caféiers. Il serait possible de multiplier les exemples. La plupart tiennent compte de l'expérience des diversifications traditionnelles et les adaptent aux exigences nouvelles, en y associant de nouvelles spéculations. Cette diversification se maintient ensuite, à un degré moindre (Tulet, 2000).

\section{LES INITIATIVES AUTOUR DU CAFÉ " DURABLE "}

L'effondrement des prix internationaux qui a suivi la fin de l'Accord international sur le café (dernière décennie $d u X X^{e} s$.) a provoqué une réaction de bon nombre de pays producteurs qui ont cherché 
7 - II n'y a pas de retour aux variétés traditionnelles, sauf cas exceptionnels. Toutefois la vogue des cafés spéciaux favorise le maintien de diverses caféières avec des variétés traditionnelles, gérées en bio, grâce à une rémunération supérieure, obtenue sous une forme contractuelle. à se démarquer d'une offre "banale " en proposant des cafés "d'origine " ou des cafés supposés disposer de qualités spécifiques. Malgré sa vogue actuelle, ce mouvement ne touche qu'une très faible part de la production, on l'estime à $1 \%$ des exportations, mais cette part augmente rapidement. Parmi ces initiatives, le café " bio " est l'une des plus anciennes et des plus importantes.

\section{Le café " bio " : la réaction dans la continuité}

Même s'il reste très largement minoritaire, l'apparition du café " biologique " constitue une évidente réaction au développement de ces nouvelles formes de production, qui entraînent toujours plus d'intensification et toujours plus de consommation d'intrants industriels. Les tenants de ce café "bio ", orgánico comme on dit en Amérique latine, reprochent à ces formes productivistes de négliger ou d'ignorer les problèmes de maintien à long terme des équilibres écologiques, auxquels les caféiculteurs dans leur majorité restent très attachés, et ceux liés à l'élévation de plus en plus forte des coûts de production. La disparition progressive des subventions aux engrais et autres produits industriels dans la plupart des pays ainsi que les périodes de baisse des prix internationaux du café ont contribué à accorder plus de poids à ces arguments (Tulet, 2000).

L'audience, la faveur même dont bénéficie actuellement le café orgánico, y compris parmi les producteurs qui ne l'adoptent pas, se nourrit aussi du fait que certaines des techniques qui lui sont associées reprennent, réactualisent, revitalisent un certain nombre de pratiques culturales jamais oubliées. D'une certaine manière, il s'agit d'un changement dans une évidente continuité, en accordant une modernité nouvelle à ce qui était autrefois effectué de façon... toute " naturelle ". II faut souligner qu'il n'est pas question pour la plupart d'accepter un retour aux rendements d'autrefois. Cela ne serait plus supportable et compromettrait la survie de l'exploitation. L'enjeu est d'approcher ou de maintenir les rendements obtenus avec les nouvelles variétés ${ }^{7}$ et les intrants industriels, au moyen de techniques évitant l'application de ces derniers. La fertilisation organique est obtenue selon les cas soit par reconversion de la pulpe de café en terreau au moyen de la lombriculture, soit par élaboration de composts, ce qui demande beaucoup de temps et d'efforts. Ceux-ci le sont d'autant plus qu'un engrais obtenu aussi difficilement doit être employé au mieux. Cela suppose parfois un remaniement des versants pour qu'ils ne soient pas emportés par les eaux de pluie.

Le modèle de base proposé est ainsi fondé sur une combinaison des systèmes de production traditionnels et des techniques de culture biologique, avec pour objectif d'optimiser les interrelations écologiques et économiques. La biodiversité maximale est recherchée, non seulement pour assurer la conservation des ressources naturelles, mais aussi pour accroître la productivité biologique et limiter les pertes en énergie et en fertilité. Elle est obtenue non seulement en favorisant les espèces arborées dotées de la meilleure productivité biologique (tronc, branches, feuillage), mais en introduisant ou en réintroduisant le plus grand nombre $\mathrm{d}$ 'autres espèces végétales, qui à leur tour permettent le développement d'une plus grande variété floristique et animale. Cette diversité permet aussi de limiter et de contrôler dans une certaine mesure les attaques effectuées par les très nombreux parasites et affections présents en milieu tropical humide.

Il existe par ailleurs un très grand nombre de caféiculteurs qui, tout en restant dans un modèle de type traditionnel, avec application d'intrants industriels, cherchent à en limiter l'usage ou adoptent même des techniques de type " bio ". On rencontre ainsi des ateliers de lombriculture destinés à recycler la pulpe des cerises de café, y compris dans les exploitations parmi les plus productivistes, à la fois pour trouver une solution au problème de cette pulpe, qui représente d'énormes quantités, mais également pour récupérer un terreau de bonne qualité à bon compte. De même, on observe une diffusion de plus en plus grande des machines destinées à limiter la consommation de l'eau dans le traitement des cerises après récolte, phénomène tout particulièrement marqué en Colombie. Beaucoup de ces adoptions relèvent d'un calcul économique bien compris, puisqu'ils aboutissent le plus souvent à une diminution des coûts de production. Cet objectif est également au moins en partie celui des producteurs " bio ". Jusqu'à une date très récente, l'engouement pour la production " écologique " a été beaucoup plus important pendant les périodes de baisse des prix internationaux. Pendant ces périodes, le différentiel de rémunération entre le café " bio " et le café " normal " est le plus élevé, ce qui représente un facteur incitatif évident. Lorsque les prix se relèvent, les efforts supplémentaires très importants que les producteurs doivent consentir pour un type de production " bio " sont alors souvent jugés trop élevés. On observe alors son abandon de la part de certains caféiculteurs. Cet abandon est d'autant plus grave qu'il faut quatre ans de culture sans intrants industriels pour que la production soit certifiée " bio ". II faut ajouter que ces évolutions conjoncturelles n'ont plus la même importance avec le développement de plus en plus grand de relations contractuelles durables.

\section{Émergence du café « durable »}

La notion générale de café " durable " a été mise au point, il y a peu, par l'industrie du café de spécialité en Amérique du Nord, même si les premières initiatives ont été européennes autour du " commerce équitable " (Daviron, Ponte, 2007, p. 213). Cette formule peut être considérée comme un moyen commode de rassembler toute une série d'initiatives aboutissant généralement à une certification commerciale. On distingue ainsi : 
- le café biologique, défini par une utilisation minimale d'intrants industriels ;

- le commerce équitable, dont les produits peuvent ne pas être "bio " (même s'ils le sont pour une large part), destiné à améliorer les conditions de vie de producteurs, grâce au renforcement des organisations de producteurs, une rémunération plus " juste " et des relations contractuelles établies dans la durée ;

- le café d'ombre, une initiative récente de certification, destinée à œuvrer pour la conservation du couvert forestier et favoriser la biodiversité animale :

- Utz Kapeh, une initiative elle aussi très récente, dont le but est d'élaborer un code de bonne conduite pour la production de café durable sur la base des " bonnes conduites agricoles" (Daviron Ponte, 2007, p. 233).

II faudrait ajouter à ces diverses certifications les initiatives qui se multiplient aujourd'hui et qui visent à définir des dénominations d'origine. Celles-ci s'appuient également sur un cahier des charges reprenant une grande partie des recommandations proposées pour le café durable. Elles visent à extraire du marché une partie de la production de café en lui conférant des qualités spécifiques qui la distinguent, qui lui donnent une identité particulière, non reproductible, qui la place en dehors de la concurrence. Dans la plupart des cas, ces initiatives n'ont pas encore abouti. Les procédures et le but poursuivis sont très proches de ceux qui régissent les appellations d'origine, assez peu prisées dans les pays du Nord de l'Europe et plus globalement dans monde anglosaxon. Est-ce une des raisons des difficultés de leur mise en application en Amérique latine ?

De fait, tout cela pour l'instant correspond d'abord à des certifications spécifiques, apposées sur le paquet de café vendu, beaucoup moins à des pratiques productives originales : le café " équitable " est " bio " le plus souvent. Par ailleurs, on ne peut guère imaginer celui-ci sans qu'il soit conduit dans le cadre d'une diversité végétale et productive, impliquant un couvert forestier, c'est-à-dire sans qu'il soit un café d'ombre. La distinction effectuée au niveau de la certification commerciale a donc peu de sens au niveau de la sphère productive. Mais elles ont toutes un point commun, celui de mettre l'accent sur l'importance des formes associatives au niveau de la production.

Ces associations, coopératives le plus souvent, rassemblent en particulier des petits producteurs qui n'auraient pu se lancer dans la culture biologique ou adopter les pratiques requises de manière contractuelle, sans disposer de garantie d'accès à des structures de commercialisation. Le développement récent des cafés "durables" résulte donc le plus souvent de la rencontre entre certaines potentialités des producteurs locaux et des initiatives militantes, sans lesquelles les premières n'auraient guère eu les moyens de s'exprimer. Les ONG de tout type s'insèrent dans ce dispositif, en assurant l'accès aux marchés internationaux et en procurant des moyens financiers. Elles établissent des relations contractuelles pluriannuelles, impliquant l'observation d'un cahier des charges qui fait la part belle au respect de l'environnement, mais pas seulement. Cela explique une géographie du commerce " durable " assez spécifique. Celui-ci a pris une place plus importante dans quelques pays particuliers, par exemple au Pérou, avec une histoire récente qui avait vu le démantèlement partiel des systèmes traditionnels de commercialisation, ou le Sud du Mexique, dans lequel beaucoup d'initiatives ont eu pour origine l'action de communautés de base animées par des membres du clergé catholique. Pour eux, le secteur de la caféiculture ne représente qu'une partie d'une action polymorphe, visant à permettre à des communautés particulièrement exploitées de trouver les moyens de s'affirmer et de mieux vivre.

Tout cela demeure encore aujourd'hui très minoritaire, malgré une croissance tout à fait significative, qui se traduit en particulier par la mise en place de lignes de produits "durables " ou "équitables ", y compris par les grandes firmes du secteur. Rançon de ce succès, le problème du contrôle de la réalité des bénéfices et de l'autonomie de décision laissée au producteur risque de se poser à terme. Dès à présent, il existe dans certains cas une réelle opacité dans I'attribution des diverses certifications. Certaines sont très coûteuses pour les producteurs. Toutefois, il semble pour l'instant évident que dans le cas des associations militantes actuelles, les productions "durables " contractuelles apportent des avantages incontestables aux producteurs insérés dans ces filières.

\section{CONCLUSION}

L'exemple de la caféiculture souligne donc la part des déterminations initiales dans la durabilité du système de production agricole. Dans le cas du Brésil, il est évident que la recherche du profit le plus immédiat et le plus élevé qui soit ne peut aboutir qu'à la destruction du milieu exploité. Ce type d'agriculture prédatrice ne relève pas du passé : on en trouve des exemples aujourd'hui au Mexique avec des entrepreneurs agricoles qui exploitent des terres louées pour du maraîchage, jusqu'à épuisement de leur fertilité et l'apparition de maladies. Inversement, dans le cas des paysanneries du café, qui forment l'essentiel des producteurs hispanophones, la fondation et l'appropriation d'un territoire prend une place très importante. Le planteur veut " s'établir ". De plus, le système de production mis en place a longtemps très largement contribué à cette durabilité, ce qui explique le maintien sur un même lieu d'une activité caféière sur de nombreuses générations.

Dans la plupart des cas, celle-ci a résisté à la transformation radicale des formes de production 
provoquées par l'adoption des nouvelles variétés à haut rendement. Cela s'est produit par le biais $\mathrm{d}^{\prime}$ adaptations parfois très importantes, dans lesquelles se mêlent des logiques productives bien comprises, mais également la volonté de maintenir une certaine autonomie et l'attachement à une production dont on tire fierté. II ne semble pas faire de doute que ces sentiments interviennent également dans la conversion (ou le retour, dans une certaine mesure) à des productions supposées plus saines et de meilleure qualité. Le sentiment d'appartenir à une culture du café spécifique, voire une civilisation, constitue un élément important dans le renouvellement d'identités sociales spécifiques.

Cette relative durabilité économique et environnementale de la caféiculture des pays hispanophones se double d'une durabilité sociale très originale : le plus souvent, lorsqu'une société de caféiculteurs s'établit dans une région, elle tend à perdurer, en dépit de toutes les épreuves qu'elle peut subir. Les crises successives du café ont paradoxalement renforcé le groupe des paysans caféiculteurs. Ces derniers, extrêmement fiers de leur savoir-faire, de ce qu'ils élaborent, sont prêts à se mobiliser pour défendre ce que certains d'entre eux n'hésitent pas à qualifier de " civilisation caféière " ! Le cas échéant, comme au Costa Rica, ils apparaissent comme les meilleurs défenseurs, les porte-drapeau d'une société qui se revendique comme " démocratique ", proche de ce monde de petits producteurs indépendants (la feuille de café fait partie des blasons de plusieurs provinces du pays). Dans d'autre cas, cette défense de la caféiculture peut également aboutir à la mise en place de régimes populistes, voire dictatoriaux : ce sont les caféiculteurs andins qui ont porté et maintenu au pouvoir les dictateurs vénézuéliens de la fin du $X I X^{e}$ et de la première partie du $X X^{e} s$. ...

\section{BIBLIOGRAPHIE}

DAVIRON B., PONTE S., 2007, Le paradoxe du café, Versailles, ed. Quae, $360 \mathrm{p}$.

DEFFONTAINES P., 1940, Geografia humana do Brasil, Rio de Janeiro, $118 \mathrm{p}$.

GRANDJEAN P., 1999, Note sur la spécificité de la caféiculture brésilienne en Amérique latine, Toulouse, Ateliers de Caravelle, n¹4, p. 107-115.
GRANDJEAN P., TULET J.-Ch., 2000, Le café, culture exemplaire du Brésil, Caravelle, Toulouse, $n^{\circ} 75$, p. 93-108.

GOUROU P., 1947, Les Pays tropicaux, Paris, $200 \mathrm{p}$.

GOUROU P., 1982, Terres de bonne espérance. Le monde tropical, Paris, Plon, $456 \mathrm{p}$.

MONBEIG P., 1952, Pionniers et Planteurs de Sao Paulo, Paris, Armand Colin, $376 \mathrm{p}$.

PALACIOS M., 1983, El café en Colombia, 18501970. Una historia económica, social y política, El Colegio de México, México, El Ancora editores, 1983, $558 \mathrm{p}$.

SALCEDO-BASTARDO J.-L., 1982, Historia fundamental de Venezuela, Caracas, UCV, ed. de la biblioteca, $652 \mathrm{p}$.

TULET J.-Ch., 1992, Le café et son cortège : les associations culturales dans la caféiculture latinoaméricaine, Toulouse, GEODOC $\mathrm{n}^{\circ} 38$, série MOCA $\mathrm{n}^{\circ} 2$, p. 12-18.

TULET J.-Ch., 1997, La prééminence des producteurs familiaux dans la caféiculture de I'Amérique hispanophone, in HAUBERT M. (dir.), Les paysans, l'État et le marché, Sociétés paysannes et développement, Paris, Public. de la Sorbonne, p. 267-278.

TULET J.-Ch., 1998, Le rôle déterminant des facteurs endogènes dans l'évolution des caféicultures latino-américaines, Tiers Monde, Paris, t. XXXIX, p. 819-833.

TULET J.-Ch, 2000, Tradition et modernité dans la culture du café biologique chez les producteurs latino-américains, in TULET J.-CH., GILARD J. (dir.), La fleur du café : Caféiculteurs de l'Amérique hispanophone, Paris, Karthala (coll. Hommes et Sociétés), p. 67-84. 